\title{
Characterization of Ovalbumin Absorption Pathways in the Rat Intestine, Including the Effects of Aspirin
}

\author{
Tomoharu Yokooji, ${ }^{a, b}$ Hitomi Nouma, ${ }^{b}$ and Hiroaki Matsuo*,a,b \\ ${ }^{a}$ Department of Pathophysiology and Therapeutics, Institute of Biomedical and Health Sciences, Hiroshima \\ University; and ${ }^{b}$ Faculty of Pharmaceutical Sciences, Hiroshima University; 1-2-3 Kasumi, Minami-ku, Hiroshima \\ 734-8553, Japan. \\ Received April 9, 2014; accepted June 1, 2014
}

\begin{abstract}
Ingested proteins are absorbed from the intestinal lumen via the paracellular and/or transcellular pathways, depending on their physicochemical properties. In this study, we investigated the absorption pathway(s) of ovalbumin (OVA), an egg white-allergen, as well as the mechanisms of aspirin-facilitated OVA absorption in rats. In situ intestinal re-circulating perfusion experiments showed that the absorption rate of fluorescein isothiocyanate (FITC)-labeled OVA in the distal intestine was higher than that for a marker of non-specific absorption, FITC-dextran (FD-40), and that colchicine, a general endocytosis inhibitor, suppressed OVA absorption. In the distal intestine, bafiromycin $A_{1}$ and phenylarsine oxide inhibited the OVA absorption rate, whereas mehyl- $\beta$-cyclodextrin exerted no significant effects. Thus, OVA is preferentially absorbed from the distal intestine via the paracellular and receptor- and clathrin-mediated endocytic pathways. Furthermore, aspirin increased OVA absorption in the presence or absence of colchicine, indicating that aspirin facilitated OVA absorption by inducing intestinal barrier disruption and paracellular permeability.
\end{abstract}

Key words ovalbumin; aspirin; clathrin-dependent endocytosis; paracellular pathway; absorption

Food-dependent exercise-induced anaphylaxis (FDEIA) is a peculiar form of food allergy induced by physical exercise in combination with causative food ingestion. ${ }^{1,2)}$ In addition to exercise, intake of non-steroidal anti-inflammatory drugs (NSAIDs), especially aspirin (ASP), is another well-documented trigger for FDEIA symptoms. ${ }^{3)}$ Patients with FDEIA typically exhibit generalized urticaria, dyspenea, and anaphylaxis induced by a type I allergic reaction. ${ }^{4-6)}$ Many kinds of foods appear to be responsible for FDEIA symptoms, including wheat, shrimp, vegetables, and eggs. ${ }^{4,5)}$ Several previous reports have shown that increased intestinal absorption of allergen is considered a key factor in the development of FDEIA symptoms. ${ }^{7-9)}$ Hanakawa et al. $^{7)}$ suggested that the symptom development depends on the amount of ingested allergen. Kohno et $a l^{8)}$ also reported that an increased serum level of gliadin, a major FDEIA allergen from wheat flour, was required to elicit allergic reactions in patients with a wheatFDEIA provocation test. Thus, it is important to elucidate the intestinal absorption mechanisms of allergens that trigger allergic reaction in patients with food allergy including FDEIA.

Orally ingested dietary proteins are not readily absorbed from the intestinal lumen in intact form owing to their enzyme digestibility and poor membrane permeability. Most ingested proteins are degraded by luminal gastric and pancreatic proteases as well as brush-border proteases, including aminopeptidases. However, a small amount of intact protein, which has escaped protease degradation, is absorbed via specialized microfold (M) cells in Peyer's patches and across epithelial cells by transcytosis (i.e., apical endocytosis and basolateral exocytosis). ${ }^{10)}$ Endocytic pathways are divided into two types: clathrin-dependent and clathrin-independent (e.g., caveolae-mediated endocytosis). ${ }^{11)}$ Intact proteins are absorbed across intestinal epithelial cells through paracellular as well as transcellular pathways. Although normal tight junctions limit absorption of macromolecules through the paracellular path-

The authors declare no conflict of interest. way, strenuous exercise and activation of intestinal mast cells enhances macromolecule permeability following impairment of the paracellular pathway. ${ }^{12,13)}$ NSAIDs also facilitate intestinal absorption of food allergens owing to reduced prostaglandin production by inhibiting cyclooxygenases (COXs). ${ }^{14,15)}$ We previously reported that the absorption rate of lysozyme, an egg-white allergen from rat proximal intestine, was higher than that from distal intestine, and ASP enhanced lysozyme absorption. ${ }^{16)}$ However, the absorption properties of ovalbumin (OVA), another important allergen in egg-allergy and eggFDEIA, ${ }^{17,18)}$ are poorly understood because macromolecule absorption pathways depend on their physicochemical properties, such as molecular size and electric charge. ${ }^{16,19)}$ OVA has a molecular mass of $45 \mathrm{kDa}$ and a $\mathrm{p} I$ of 4.7 , and belongs to the serine-protease inhibitor family. ${ }^{20,21)}$ Although it has been shown that intact OVA is absorbed into peripheral blood and lymph from the gastrointestinal lumen, ${ }^{22,23)}$ how ASP effects OVA absorption pathways may be different from that of lysozyme, with a molecular mass of $14 \mathrm{kDa}$ and $\mathrm{p} I$ of $11 .^{24)} \mathrm{In}$ this study, we investigate OVA absorption pathway(s), and the effects of ASP on OVA absorption in rat intestine.

\section{MATERIALS AND METHODS}

Materials OVA (Grade V), bovine serum albumin (BSA; Cohn fraction V), fluorescein isothiocyanate (FITC), FITC-dextran40 (FD-40; average molecular weight, $40 \mathrm{kDa}$ ), and protease inhibitor cocktail were purchased from SigmaAldrich (St. Louis, MO, U.S.A.). ASP, phenylarsine oxide (PAO), and methyl- $\beta$-cyclodextrin (MCD) were purchased from Wako Pure Chemical Industries, Ltd. (Osaka, Japan). Colchicine (COL) and bafilomycin $\mathrm{A}_{1}(\mathrm{BAF})$ were obtained from Nacalai Tesque (Kyoto, Japan) and LC laboratories (Woburn, MA, U.S.A.), respectively. All chemicals used were of the highest purity available.

Animals Male Sprague-Dawley (SD) rats aged 7-8 weeks were obtained from Japan SLC, Inc. (Shizuoka, Japan). Rats 
were fed a standard laboratory diet and water ad libitum for more than 1 week prior to the experiments. Experiments involving animals were carried out in accordance with the Guide for Animal Experimentation from the Committee of Research Facilities for Laboratory Animal Sciences of Hiroshima University (Hiroshima, Japan).

Preparation of FITC-Labeled OVA Labeling of OVA with FITC was undertaken as described previously. ${ }^{16)}$ Briefly, $1 \mathrm{mg}$ FITC and $100 \mathrm{mg}$ OVA were dissolved in $0.1 \mathrm{~m}$ borate buffer ( $\mathrm{pH}$ 9.0). After incubation for $3 \mathrm{~h}$ at room temperature, $\mathrm{pH}$ was adjusted to 7.5 with $0.1 \mathrm{~m}$ boric acid. The reaction mixture was subjected to gel filtration using the Superdex HiLoad 26/600 75 prep grade column (GE Healthcare, Little Chalfont, U.K.) to remove free FITC. The solution collected was dialyzed using cellulose membranes with a molecularweight cutoff of $3.5 \mathrm{kDa}$ overnight at $4{ }^{\circ} \mathrm{C}$ and concentrated by freeze-drying. When the lyophilized proteins were subjected to sodium dodecyl sulfate-polyacrylamide gel electrophoresis (SDS-PAGE), a single band was observed by Coomassie brilliant blue (CBB) staining and imaging with a Fluoroimage Analyzer (Typhoon FLA-7000, GE Healthcare, Little Chalfont, U.K.). The band size was the same as that with authentic OVA $(45 \mathrm{kDa})$, suggesting no degradation of OVA during FITC labeling.

In Situ Re-circulating Perfusion Study of FITC-OVA The intestinal absorption of FITC-OVA was evaluated in a re-circulating perfusion manner as described previously, with slight modifications. ${ }^{16)}$ Briefly, rats were fasted overnight and anesthetized with pentobarbital $(30 \mathrm{mg} / \mathrm{kg}$, intraperitoneally (i.p.)). The proximal (a $20-\mathrm{cm}$-long segment from $5-\mathrm{cm}$ below the opening of the bile duct) and distal small intestine (a 20-cm-long segment from the ileocecum) were used to elucidate regional differences in FITC-OVA absorption. Proximal and distal segments were pre-perfused with phosphate-buffered saline (PBS) at $\mathrm{pH} 6.5$ and 7.4, respectively and warmed at $37^{\circ} \mathrm{C}$ for $30 \mathrm{~min}$. Each intestinal segment was then perfused in a re-circulating manner at $1 \mathrm{~mL} / \mathrm{min}$ with $10 \mathrm{~mL}$ of each type of PBS containing FITC-OVA $(0.4 \mu \mathrm{M})$. Dimethyl sulfoxide (DMSO, $1 \%(\mathrm{v} / \mathrm{v}))$ and protease inhibitors [4-(2-aminoethyl)benzenesulfonyl fluoride $(1.04 \mathrm{~mm})$, aprotinin $(0.80 \mu \mathrm{M})$, bestatin $(40 \mu \mathrm{M})$, E-64 $(14 \mu \mathrm{M})$, leupeptin $(20 \mu \mathrm{M})$ and pepstatin A $(15 \mu \mathrm{M})]$ were added to increase the solubility of modulators, and to prevent OVA degradation in intestinal perfusate. To examine the effects of modulators, unlabeled OVA $(0.4-100 \mu \mathrm{M})$, BSA $(100 \mu \mathrm{M})$, COL $(100 \mu \mathrm{M})$, BAF $(100 \mathrm{~nm})$, PAO $(10 \mu \mathrm{M}), \operatorname{MCD}(5 \mathrm{~mm})$ and ASP $(16.7 \mathrm{~mm})$ were added to perfusate. BAF and PAO were used only in the pre-perfusate. The intestinal perfusate $(200 \mu \mathrm{L})$ was collected periodically to determine FITC-OVA concentrations. The FITC-OVA absorption rate was estimated from the disappearance rate by measuring the FITC-OVA remaining in the perfusate between 0.5 and $1.5 \mathrm{~h}$. The absorption rate of FD-40 $(0.4 \mu \mathrm{M})$ was estimated in the same way as for FITC-OVA. Perfusate concentrations of FITC-OVA and FD-40 were determined at a wavelength of $500 \mathrm{~nm}$ for excitation and $520 \mathrm{~nm}$ for emission using a Microplate Fluorometer (PerkinElmer, Inc., Waltham, MA, U.S.A.).

The half-maximal inhibitory concentration $\left(\mathrm{IC}_{50}\right)$ of unlabeled OVA and the FITC-OVA absorption rates were estimated using the Hill equation as described previously ${ }^{25)}$ :

$$
v-v_{o}=\left(v^{\prime}-v_{o}\right) /\left[1+\left([I] / \mathrm{IC}_{50}\right)^{\gamma}\right],
$$

where $v$ is the observed FITC-OVA absorption; and $v^{\prime}$ and $v_{o}$ are FITC-OVA absorption rates in the absence and presence of unlabeled OVA $(100 \mu \mathrm{M})$, respectively; $[I]$ is the initial concentration of unlabeled OVA, and $\gamma$ is the Hill coefficient. Curve fitting analysis was performed using KaleidaGraph ${ }^{\mathrm{TM}}$ program (Version 3.501, Synergy Software, Reading, PA, U.S.A.).

Stability of Intact FITC-OVA in Perfusate Perfusate FITC-OVA intactness was evaluated using Fluoroimage Analyzer FLA-7000 after separation by SDS-PAGE, as reported previously. ${ }^{16)}$ Briefly, perfusate was added in loading buffer that comprised $65 \mathrm{~mm}$ Tris- $\mathrm{HCl}, 10 \%(\mathrm{v} / \mathrm{v})$ glycerol, $5 \%(\mathrm{v} / \mathrm{v})$ 2-mercaptoethanol, 3\% SDS, and $0.01 \%$ bromophenol blue. Sample was applied to each lane of a $12.5 \%$ polyacrylamide gel, and electrophoresed. Fractioned proteins were visualized by $\mathrm{CBB}$ staining and a Fluoroimage Analyzer. Images were analyzed using ImageQuant TL software (GE Healthcare, Piscataway, NJ, U.S.A.).

Absorption of OVA after Oral Administration Rats were fasted overnight and anesthetized with pentobarbital $(30 \mathrm{mg} / \mathrm{kg}$, i.p.), and cannulated with polyethylene tubing (PE-50) at the femoral artery for blood sampling. Vehicle alone (PBS at $\mathrm{pH} 7.4)$ or ASP $(167 \mu \mathrm{mol} / \mathrm{kg})$ were administered orally using a stainless steel feeding tube. OVA $(1.1 \mu \mathrm{mol} / \mathrm{kg})$ dissolved in PBS was administered orally $30 \mathrm{~min}$ after the treatment. Blood $(0.25 \mathrm{~mL}$ each) was collected at a designated time interval for $3 \mathrm{~h}$ via the cannula inserted at the femoral artery, and centrifuged to collect plasma. To estimate the extent of absorption, OVA was injected intravenously at a dose of $1.0 \mathrm{nmol} / \mathrm{kg}$ via the femoral vein. Blood was then collected from the femoral artery periodically for $3 \mathrm{~h}$ to measure plasma OVA concentrations, which were determined by a sandwich enzyme-linked immunosorbent assay kit (Morinaga Institute of Biological Science, Yokohama, Japan) using OVA as a standard. The detection limit of OVA was 2.2 to $556 \mathrm{pm}$ under these conditions. The extent of oral OVA absorption was estimated as the absorbed fraction of oral OVA $\left(F A_{\text {p.o. }}\right)$ by comparing the OVA area under the plasma concentration-time curve $(A U C)$ after oral administration, with the OVA $A U C$ after intravenous bolus injection using the following equation:

$$
F A_{\text {p.o. }}=A U C_{\text {p.o. }} / A U C_{\text {i.v. }} \times D_{\text {i.v. }} / D_{\text {p.o. }}
$$

where $A U C_{\text {p.o. }}$ and $A U C_{\text {i.v. }}$ are $A U C$ s of OVA after oral and intravenous administration, respectively; $D_{\text {p.o. }}$ and $D_{\text {i.v. }}$ are the OVA doses in the respective experiments.

Statistical Analyses Data are displayed as the mean \pm standard error of the mean (S.E.). Mean value differences between groups were assessed by Kruskal-Wallis or ANOVA tests, followed by a post hoc Tukey or Student's $t$-test. A $p<0.05$ was considered significant.

\section{RESULTS}

Characteristics of Absorption Pathway(s) of FITC-OVA in Rat Intestine Intestinal FITC-OVA absorption was examined by an in situ re-circulating perfusion method. The FITC-OVA absorption rate was $\approx 1$.7-fold greater in the distal intestine than in the proximal intestine (Fig. 1A). The FITCOVA absorption rate in the distal intestine was also 2.3-fold greater than that of FD-40, a marker for non-specific pathways (i.e., paracellular and fluid-phase endocytic pathways), and decreased by $\approx 56 \%$ following treatment with COL (a general in- 
(A)

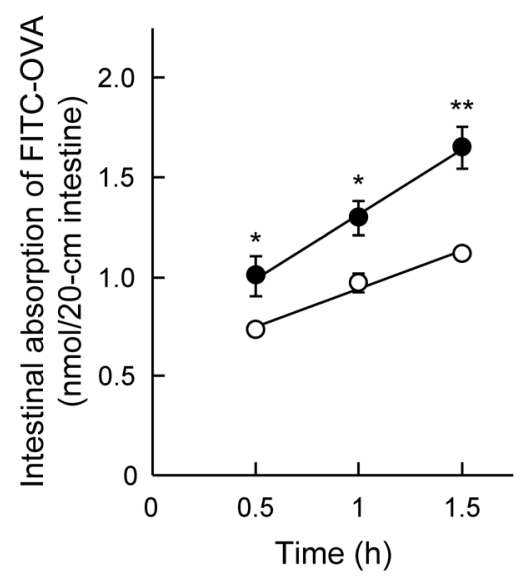

(C)

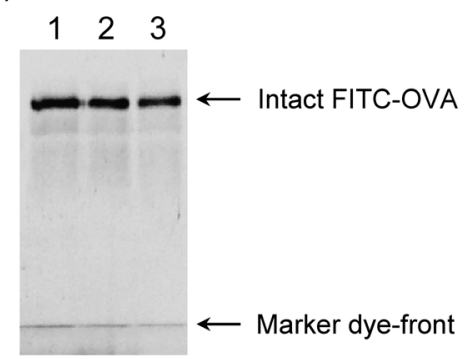

(B)

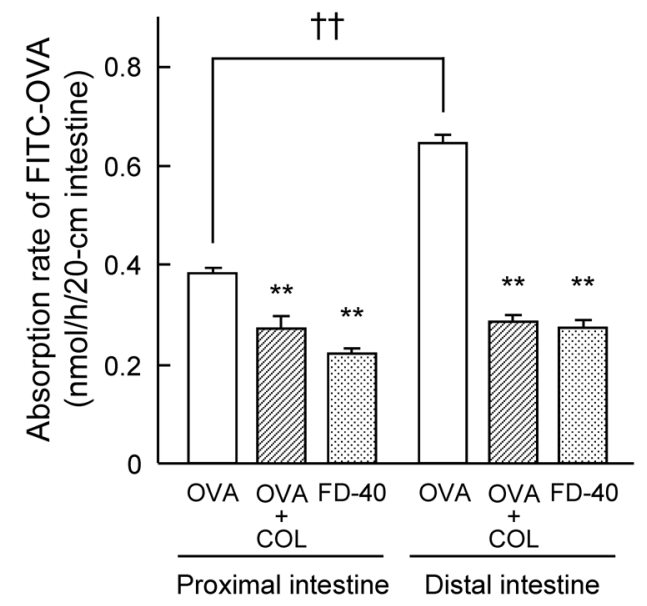

Fig. 1. Time-Course for FITC-OVA Intestinal Absorption (A) and FITC-OVA and FD-40 Absorption Rates in the Absence or Presence of COL (B) as Evaluated by in Situ Re-circulating Perfusion Using the Proximal and Distal Intestines, and Intactness of FITC-OVA in the Intestinal Perfusate (C)

The amounts of FITC-OVA and FD-40 absorbed were assumed to be of the same magnitude as the amount that was eliminated from the perfusate. (A) Open and closed circles represent the proximal and distal intestine, respectively. Each value represents the mean \pm S.E. for four rats. $* p<0.05$ and $* * p<0.01$ : significantly different from proximal intestine. (B) COL concentration in perfusate was $100 \mu \mathrm{M}$. Each value represents the mean \pm S.E. for four rats. $* * p<0.01$ and ${ }^{\dagger} p<0.01$ : significantly different from control (OVA) and proximal intestine, respectively. (C) FITC-OVA intactness in intestinal perfusate, obtained at 90 min, evaluated by a Fluoroimage Analyzer after SDS-PAGE. Lane 1 is intact FITC-OVA, and lanes 2 and 3 are perfusate from proximal and distal intestine, respectively.

(A)

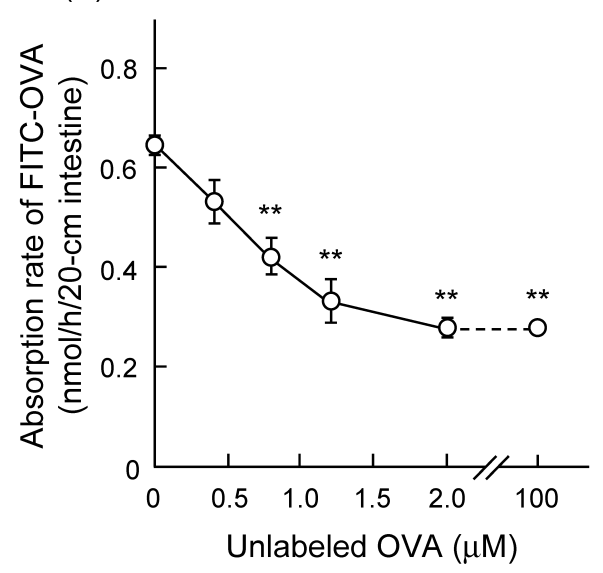

(B)

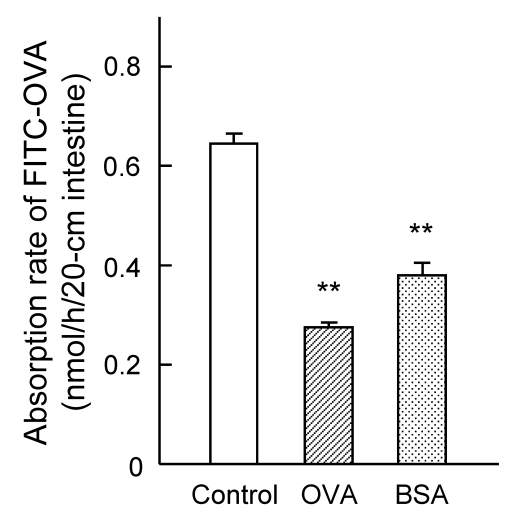

Fig. 2. Effects of Unlabeled OVA (A) and BSA (B) on FITC-OVA Intestinal Absorption in the in Situ Re-circulating Perfusion of the Distal Intestine

The amount of FITC-OVA absorbed was assumed to be of the same magnitude as the amount that was eliminated from the perfusate. (A) The absorption rate of FITCOVA was evaluated in the absence (control) or presence of various concentrations of unlabeled OVA. (B) Concentrations of both unlabeled OVA and BSA in the perfusate were $100 \mu \mathrm{M}$. Each value represents the mean \pm S.E. for four rats. $* * p<0.01$ : significantly different from control.

hibitor of endocytosis), relative to that seen with controls (Fig. 1B). These results indicate that both the paracellular pathway and specific-endocytosis mechanism(s), including adsorptive and/or receptor-mediated endocytosis, may be involved in FITC-OVA absorption in the distal intestine. The FITC-OVA absorption rate in the proximal intestine was 1.7-fold higher than that of FD-40, and was affected slightly by COL (Fig.
1B). In the perfusate samples, $90 \mathrm{~min}$ after re-circulating perfusion, intact FITC-OVA was observed, suggesting that FITCOVA was essentially stable in the intestinal perfusate during the time course of the perfusion (Fig. 1C).

Characteristics of Endocytic Pathway(s) of FITC-OVA in Rat Intestine We examined the effects of various concentrations of unlabeled OVA and BSA on the absorption of 
FITC-OVA in the distal intestine to characterize the specificity of FITC-OVA intestinal absorption. As shown in Fig. 2A, intestinal absorption of FITC-OVA was inhibited by unlabeled OVA in a concentration-dependent manner. The specific (endocytic) absorption pathway of FITC-OVA appeared to be inhibited completely in the presence of $2 \mu \mathrm{M}$ of unlabeled OVA, because the absorption rate decreased to the same level as seen for COL treatment and the absorption rate of FD-40 as shown in Fig. 1B. The $\mathrm{IC}_{50}$ value of unlabeled OVA was $0.61 \mu \mathrm{M} \quad(\gamma=2.30)$. Notably, the FITC-OVA absorption rate decreased in the presence of BSA to almost the same level as seen for unlabeled OVA treatment (Fig. 2B).

To further elucidate the molecular mechanisms underlying the endocytic pathways of FITC-OVA absorption, the effects of BAF (an inhibitor of endosomal acidification), PAO (an inhibitor of clathrin-dependent endocytosis) and MCD (an inhibitor of caveolae-mediated endocytosis) on FITC-OVA absorption were evaluated (Fig. 3). The FITC-OVA absorption rate was significantly inhibited by pretreatment with BAF or PAO, decreasing to the same level seen in COL treatment. In contrast, no significant inhibitory effect was observed for MCD.

Effect of ASP on Intestinal Absorption Rate of FITCOVA The effects of clinical doses $(16.7 \mathrm{~mm})$ of ASP on FITC-OVA intestinal absorption were evaluated by an in situ re-circulating perfusion method (Fig. 4). ASP increased the FITC-OVA absorption rate by approximately 2.0 - and 1.7-fold more than that in the proximal and distal intestine, respectively. In COL-treated rats, ASP increased the FITC-OVA absorption rate in both intestinal regions. Thus, ASP modulates intestinal OVA absorption through a paracellular but not endocytic pathway.

Effect of ASP on Oral Absorption of OVA OVA given intravenously disappeared from plasma rapidly, following a first order rate constant (Fig. 5A). The relevant biological and kinetic parameters are shown in Table 1 . The apparent distribution volume of OVA was $\approx 170-210 \mathrm{~mL} / \mathrm{kg}$ body weight, which corresponds to the extracellular space volume (Table
1). ${ }^{26)}$ Next, we sought to evaluate the ASP-facilitated absorption of OVA into blood by examining the effect of ASP on OVA intestinal absorption following oral administration (Fig. 5B). Oral OVA was absorbed gradually with time, and plasma concentrations displayed flip-flop kinetics, indicating that the elimination rate was greater than the absorption rate (Table 1). Plasma kinetics showed that ASP treatment increased OVA oral absorption ( $F A_{\text {p.o }}$ values) by $\approx 2.6$-fold compared with controls $\left(F A_{\text {p.o. }}\right.$ : control, $0.00158 \pm 0.00047 \%$; ASP-treated rats, $0.00404 \pm 0.00030 \%, * * p<0.01)$.

\section{DISCUSSION}

In this study, we investigated the absorption pathway(s) and in vivo kinetics of OVA, and the effects of ASP on OVA absorption in rat intestine. FITC-OVA, with a molecular mass

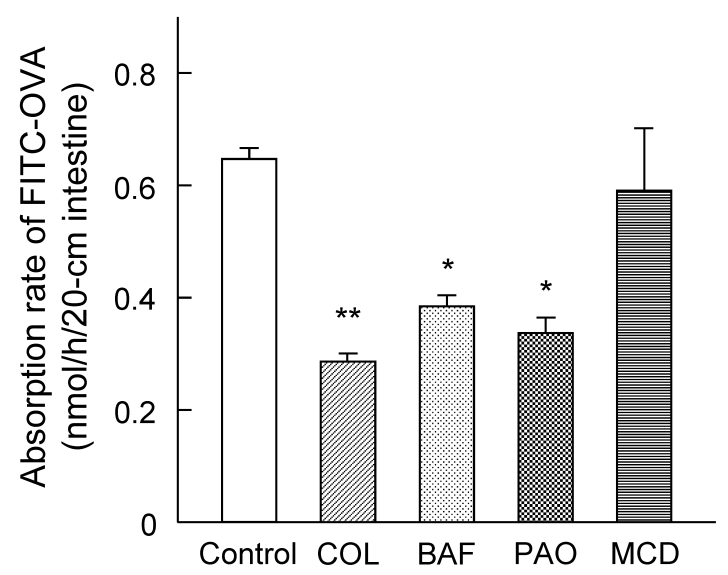

Fig. 3. Effect of Endocytosis Inhibitors on FITC-OVA Intestinal Absorption in the in Situ Re-circulating Perfusion of Distal Intestine

The amount of FITC-OVA absorbed was assumed to be of the same magnitude as the amount that was eliminated from perfusate. Concentrations of COL, BAF, $\mathrm{PAO}$, and MCD in the perfusate were $100 \mu \mathrm{M}, 100 \mathrm{~nm}, 10 \mu \mathrm{M}$, and $5 \mathrm{~mm}$, respectively. To evaluate the effect of BAF and PAO, they were added only in the pre-perfusate. Each value represents the mean \pm S.E. for four rats. $* * p<0.01$ and $* p<0.05$ : significantly different from control.
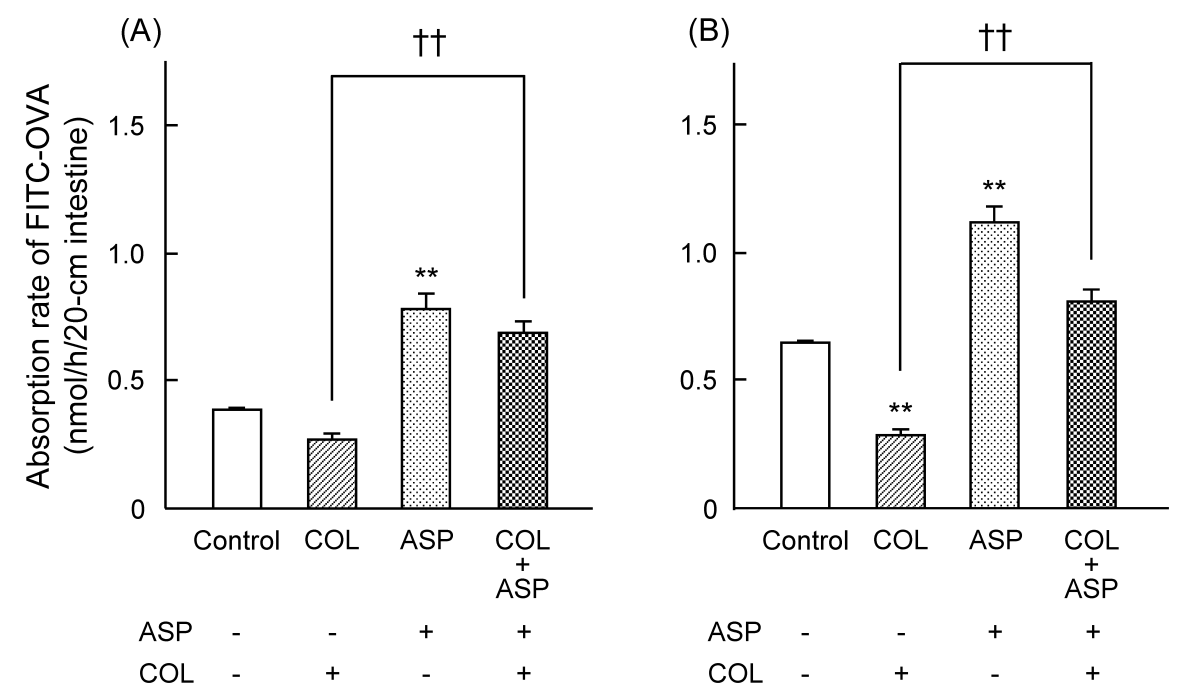

Fig. 4. Effect of ASP on FITC-OVA Intestinal Absorption in the in Situ Re-circulating Perfusion Study Using the Proximal (A) and Distal Intestine (B)

The amount of FITC-OVA absorbed was assumed to be of the same magnitude as the amount that was eliminated from perfusate. Concentrations of COL and ASP in the perfusate were $100 \mu \mathrm{M}$ and $16.7 \mathrm{~mm}$, respectively. Each value represents the mean \pm S.E. for four rats. $* * p<0.01$ and ${ }^{\dagger \dagger} p<0.01$ : significantly different from for control and COL treatment, respectively. 
(A)

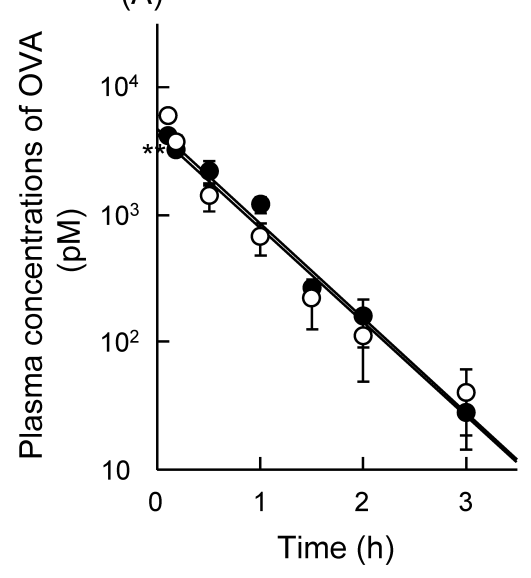

(B)

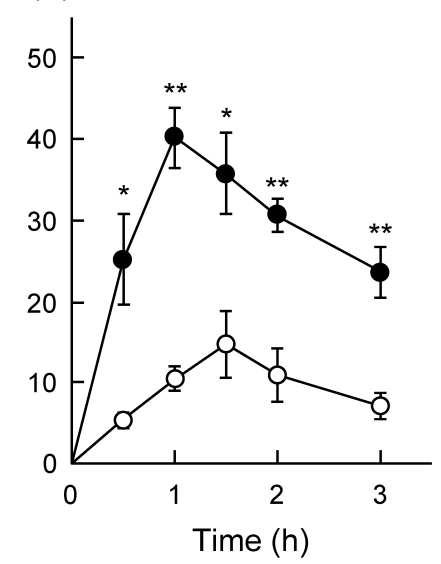

Fig. 5. ASP Effect on OVA Plasma Concentrations after Intravenous (A) and Oral (B) Administration

ASP was administered at a dose of $167 \mu \mathrm{mol} / \mathrm{kg} 30 \mathrm{~min}$ before intravenous $(1.0 \mathrm{nmol} / \mathrm{kg})$ or oral $(1.1 \mu \mathrm{mol} / \mathrm{kg})$ administration. Open and closed circles represent the control and ASP treatment, respectively. Each value represents the mean \pm S.E. for four rats. $* p<0.05, * * p<0.01$ : significantly different from control.

Table 1. Effect of ASP on OVA Biological Kinetic Parameters after Intravenous and Oral Administration in Rats

\begin{tabular}{|c|c|c|c|c|}
\hline & \multicolumn{2}{|c|}{ Intravenous } & \multicolumn{2}{|c|}{ Oral } \\
\hline & Control & $+\mathrm{ASP}$ & Control & $+\mathrm{ASP}$ \\
\hline Dose (nmol/kg) & 1.0 & 1.0 & 1100 & 1100 \\
\hline$A U C(\mathrm{pmol} \mathrm{h} / \mathrm{L})$ & $2518 \pm 253$ & $2690 \pm 283$ & $43.9 \pm 12.9$ & $119.5 \pm 8.8^{* *}$ \\
\hline$V_{\mathrm{d}}(\mathrm{mL} / \mathrm{kg})$ & $169.1 \pm 21.2$ & $214.0 \pm 21.7$ & n.d. & n.d. \\
\hline$k_{\mathrm{e}}\left(\mathrm{h}^{-1}\right)$ & $2.62 \pm 0.56$ & $1.83 \pm 0.20$ & n.d. & n.d. \\
\hline$T_{1 / 2}(\mathrm{~h})$ & $0.30 \pm 0.07$ & $0.39 \pm 0.04$ & n.d. & n.d. \\
\hline$C L_{\text {total }}(\mathrm{L} / \mathrm{h} / \mathrm{kg})$ & $0.41 \pm 0.04$ & $0.39 \pm 0.05$ & n.d. & n.d. \\
\hline$k_{\mathrm{a}}\left(\mathrm{h}^{-1}\right)$ & & & $0.59 \pm 0.12$ & $0.74 \pm 0.12$ \\
\hline$F A_{\text {p.o. }}\left(\times 10^{-3} \%\right)$ & & & $1.58 \pm 0.47$ & $4.04 \pm 0.30 * *$ \\
\hline
\end{tabular}

$A U C$, area under the plasma concentration-time curve; $V_{\mathrm{d}}$, apparent distribution volume; $T_{1 / 2}$, biological half-life; $C L_{\text {total }}$, total plasma clearance; $F A_{\text {p.o. }}$, absorbed fraction of oral OVA; +ASP, ASP treatment; n.d.; not determined. ASP was orally administered at $167 \mu$ mol/kg at 30 min before OVA administration. Parameters were estimated by a 1-compartment model $\left(C=C_{0} \times \mathrm{e}^{-k_{\mathrm{e}} t}\right)$ using the following equations: $V_{\mathrm{d}}=C_{\mathrm{o}} / D_{i, v}, T_{1 / 2}=0.693 / \mathrm{ke}, C L_{\text {total }}=D_{\mathrm{i} . \mathrm{v}} / A U C_{\mathrm{ivv}}$, where " $C$," " $C_{0}$, ," $k_{\mathrm{e}}$ " and " $t$ " are "plasma concentration of OVA," "initial plasma concentration of OVA," "elimination rate constant" and "time after administration of OVA," respectively. The absorption rate constant " $k$," was estimated from the slope of terminal plasma OVA concentrations after oral administration. $F A_{\text {p.o. }}$ was estimated by the equations as described in Materials and Methods. Each value represents the mean \pm S.E. for four rats. ${ }^{*} p<0.01$ : significantly different from controls.

of $45 \mathrm{kDa}$, has a molecular size similar to that of FD-40, which is absorbed from the intestinal lumen via a non-specific pathway. However, the FITC-OVA absorption rate was higher than that of FD-40 in the distal intestine, and was inhibited by COL and unlabeled OVA in a concentration-dependent manner (Figs. 1A, 2). These results indicate that FITC-OVA is absorbed via non-specific and specific pathways in the distal intestine. The inhibitory effect of BAF on FITC-OVA absorption was also observed in the distal intestine (Fig. 3). BAF is a potent inhibitor of vacuolar $\mathrm{H}^{+}$-ATPase that localizes in the endosomal membrane. The acidification of endosomes by the vacuolar $\mathrm{H}^{+}$-ATPase is an essential process for the dissociation of ligands and receptors during receptor-mediated endocytosis. ${ }^{27)}$ Thus, FITC-OVA is absorbed via receptor-mediated endocytosis in the distal intestine. To characterize the receptor ligand specificity responsible for FITC-OVA absorption, we examined the effect of BSA on FITC-OVA absorption. As shown in Fig. 2B, BSA inhibited the absorption of FITCOVA, suggesting that the receptor(s) involved in FITC-OVA absorption in the distal intestine also recognize BSA. Several studies have reported that BSA is recognized as a ligand by the megalin-cubilin complex receptor, which is expressed predominantly in the distal intestine. ${ }^{28,29)}$ Thus, there is a possibility that OVA is absorbed from the distal intestine by megalin-cubilin-mediated endocytosis.

We therefore examined the possibility that the endocytic pathway of FITC-OVA in the distal intestine may be involved. PAO inhibits clathrin-dependent endocytosis by reacting with vicinal sulfhydryls to form stable ring structures. ${ }^{30)}$ The absorption of FITC-OVA was inhibited by PAO in the distal intestine, indicating that clathrin-dependent endocytosis is involved in FITC-OVA absorption in the distal intestine (Fig. 3). Several clathrin-independent endocytic pathways have been reported, including caveolae-dependent endocytosis. ${ }^{11,31)} \mathrm{We}$ found that MCD, which disrupts caveolae integrity by cholesterol sequestration, did not affect FITC-OVA absorption in the distal intestine (Fig. 3). Thus, clathrin-dependent, but not caveolae-dependent endocytosis is involved in the FITC-OVA absorption in rat distal intestine. In addition to megalin-cubilin, the gp18, gp30 and gp60 were also identified as endocytic receptors for albumin in endothelial cells. ${ }^{32,33)}$ However, the characteristics of intestinal OVA absorption observed in this study cannot be explained by these receptors because these receptors-mediated endocytosis of albumin are reportedly caveolae-dependent pathway in contrast to our results ${ }^{33)}$ (Fig. 3).

Takano et al. ${ }^{24)}$ reported that lysozyme was also absorbed 
by clathrin-dependent endocytosis. However, the lysozyme absorption rate in the proximal intestine was higher than in the distal intestine, which is opposite to that observed for OVA absorption. ${ }^{16)}$ That is, a higher FITC-OVA absorption rate was observed in the distal intestine compared with that in the proximal intestine (Fig. 1A). Because we used protease inhibitors to evaluate the absorption of intact OVA, and lysozyme was stable in the perfusion experiments without protease inhibitors, regional differences in the absorption rates between FITC-OVA and lysozyme were thought to be independent of their degradation (Fig. 1C). Thus, OVA intestinal absorption mechanisms are different from those of lysozyme.

We previously reported that ASP enhanced the intestinal absorption of lysozyme and FITC-dextrans with different molecular weight (FD-10, average molecular weight, $9.4 \mathrm{kDa}$; FD-150, average molecular weight, $167 \mathrm{kDa}$ ) without affecting the intestinal accumulation by impairment of the enterocytic tight junction. ${ }^{16)}$ In this study, ASP increased the extent of oral absorption of OVA in the presence or absence of COL (Fig. 4). Moreover, Sugita et al. ${ }^{34)}$ showed that oral ingestion of spermine increased OVA absorption by modulating the paracellular pathway. These results strongly indicate that ASP facilitates the paracellular pathway and allergen absorption, and that augmentation is not affected by allergen properties such as molecular size and electric charge.

In conclusion, we show that intestinal OVA absorption exhibits regional differences in the rat intestine, with OVA preferentially absorbed from the distal intestine by paracellular and clathrin-dependent endocytic pathways. In addition, ASP facilitated FITC-OVA intestinal absorption via the paracellular pathway. Although further studies are necessary to identify the receptors underlying OVA intestinal absorption, these findings shed new light on the pathophysiological mechanisms of FDEIA and common food allergies.

Acknowledgment This work was supported in part by a Grant-in-Aid for Scientific Research from the Ministry of Education, Culture, Sports, Science and Technology of Japan (Grant No. 24790157).

\section{REFERENCES}

1) Kidd JM 3rd, Cohen SH, Sosman AJ, Fink JN. Food-dependent exercise-induced anaphylaxis. J. Allergy Clin. Immunol., 71, 407-411 (1983).

2) Morita E, Matsuo H, Chinuki Y, Takahashi H, Dahlström J, Tanaka A. Food-dependent exercise-induced anaphylaxis-importance of omega-5 gliadin and HMW-glutenin as causative antigens for wheat-dependent exercise-induced anaphylaxis-. Allergol. Int., 58, 493-498 (2009).

3) Matsuo H, Morimoto K, Akaki T, Kaneko S, Kusatake K, Kuroda T, Niihara H, Hide M, Morita E. Exercise and aspirin increase levels of circulating gliadin peptides in patients with wheat-dependent exercise-induced anaphylaxis. Clin. Exp. Allergy, 35, 461-466 (2005).

4) Morita E, Chinuki Y, Takahashi H. Recent advances of in vitro tests for the diagnosis of food-dependent exercise-induced anaphylaxis. J. Dermatol. Sci., 71, 155-159 (2013).

5) Morita E, Kunie K, Matsuo H. Food-dependent exercise-induced anaphylaxis. J. Dermatol. Sci., 47, 109-117 (2007).

6) Palosuo K. Update on wheat hypersensitivity. Curr. Opin. Allergy Clin. Immunol., 3, 205-209 (2003).
7) Hanakawa Y, Tohyama M, Shirakata Y, Murakami S, Hashimoto K. Food-dependent exercise-induced anaphylaxis: a case related to the amount of food allergen ingested. Br. J. Dermatol., 138, 898-900 (1998).

8) Kohno K, Matsuo H, Takahashi H, Niihara H, Chinuki Y, Kaneko S, Honjoh T, Horikawa T, Mihara S, Morita E. Serum gliadin monitoring extracts patients with false negative results in challenge tests for the diagnosis of wheat-dependent exercise-induced anaphylaxis. Allergol. Int., 62, 229-238 (2013).

9) Matsuo H, Kaneko S, Tsujino Y, Honda S, Kohno K, Takahashi H, Mihara S, Hide M, Aburatani K, Honjoh T, Morita E. Effects of non-steroidal anti-inflammatory drugs (NSAIDs) on serum allergen levels after wheat ingestion. J. Dermatol. Sci., 53, 241-243 (2009).

10) Perrier C, Corthésy B. Gut permeability and food allergies. Clin. Exp. Allergy, 41, 20-28 (2011).

11) Mayor S, Pagano RE. Pathways of clathrin-independent endocytosis. Nat. Rev. Mol. Cell Biol., 8, 603-612 (2007).

12) Yano H, Kato Y, Matsuda T. Acute exercise induces gastrointestinal leakage of allergen in lysozyme-sensitized mice. Eur. J. Appl. Physiol., 87, 358-364 (2002).

13) Pals KL, Chang RT, Ryan AJ, Gisolfi CV. Effect of running intensity on intestinal permeability. J. Appl. Physiol., 82, 571-576 (1997).

14) Kawai S. Cyclooxygenase selectivity and the risk of gastro-intestinal complications of various non-steroidal anti-inflammatory drugs: a clinical consideration. Inflamm. Res., 47 (Suppl 2), S102-S106 (1998).

15) Tachecí I, Kopácová M, Rejchrt S, Bures J. Non-steroidal anti-inflammatory drug induced injury to the small intestine. Acta Medica (Hradec Kralove), 53, 3-11 (2010).

16) Yokooji $T$, Hamura $H$, Matsuo H. Intestinal absorption of lysozyme, an egg-white allergen, in rats: kinetics and effect of NSAIDs. Biochem. Biophys. Res. Commun., 438, 61-65 (2013).

17) Caubet JC, Bencharitiwong R, Moshier E, Godbold JH, Sampson HA, Nowak-Wegrzyn A. Significance of ovomucoid- and ovalbumin-specific IgE/IgG(4) ratios in egg allergy. J. Allergy Clin. Immunol., 129, 739-747 (2012).

18) Asero R, Mistrello G, Roncarolo D, Antoniotti P, Falagiani P. Exercise-induced egg anaphylaxis. Allergy, 52, 687-689 (1997).

19) Nishikawa M, Hasegawa S, Yamashita F, Takakura Y, Hashida M. Electrical charge on protein regulates its absorption from the rat small intestine. Am. J. Physiol. Gastrointest. Liver Physiol., 282, G711-G719 (2002).

20) Hunt LT, Dayhoff MO. A surprising new protein superfamily containing ovalbumin, antithrombin-III, and alpha 1-proteinase inhibitor. Biochem. Biophys. Res. Commun., 95, 864-871 (1980).

21) Legaz ME, Pedrosa MM. Effect of polyamines on the separation of ovalbumin glycoforms by capillary electrophoresis. J. Chromatogr. A, 719, 159-170 (1996).

22) Tsume Y, Taki Y, Sakane T, Nadai T, Sezaki H, Watabe K, Kohno T, Yamashita S. Quantitative evaluation of the gastrointestinal absorption of protein into the blood and lymph circulation. Biol. Pharm. Bull., 19, 1332-1337 (1996).

23) Matsubara T, Aoki N, Honjoh T, Mizumachi K, Kurisaki J, Okajima T, Nadano D, Matsuda T. Absorption, migration and kinetics in peripheral blood of orally administered ovalbumin in a mouse model. Biosci. Biotechnol. Biochem., 72, 2555-2565 (2008).

24) Takano M, Koyama Y, Nishikawa H, Murakami T, Yumoto R. Segment-selective absorption of lysozyme in the intestine. Eur. J. Pharmacol., 502, 149-155 (2004).

25) Yokooji T, Kida M, Mori M, Akashi H, Mori N, Yoshihara S, Murakami T. Interaction of Rhei Rhizoma extract with cytochrome P450 3A and efflux transporters in rats. Pharmazie, 65, 367-374 (2010).

26) Tsuji A, Terasaki T, Imaeda N, Nishide K, Nakashima E. Effect of extracellular water volume on the distribution kinetics of betalactam antibiotics as a function of age. J. Pharmacobiodyn., 8, 167-174 (1985). 
27) Nelson N, Harvey WR. Vacuolar and plasma membrane proton adenosine triphosphatases. Physiol. Rev., 79, 361-385 (1999).

28) Christensen EI, Birn H. Megalin and cubilin: multifunctional endocytic receptors. Nat. Rev. Mol. Cell Biol., 3, 256-266 (2002).

29) Levine JS, Allen RH, Alpers DH, Seetharam B. Immunocytochemical localization of the intrinsic factor-cobalamin receptor in dogileum: distribution of intracellular receptor during cell maturation. J. Cell Biol., 98, 1111-1118 (1984).

30) Visser CC, Stevanović S, Heleen Voorwinden L, Gaillard PJ, Crommelin DJ, Danhof M, De Boer AG. Validation of the transferrin receptor for drug targeting to brain capillary endothelial cells in vitro. J. Drug Target., 12, 145-150 (2004).

31) Conner SD, Schmid SL. Regulated portals of entry into the cell.
Nature, 422, 37-44 (2003).

32) Bito R, Hino S, Baba A, Tanaka M, Watabe H, Kawabata H. Degradation of oxidative stress-induced denatured albumin in rat liver endothelial cells. Am. J. Physiol. Cell Physiol., 289, C531-C542 (2005).

33) John TA, Vogel SM, Tiruppathi C, Malik AB, Minshall RD. Quantitative analysis of albumin uptake and transport in the rat microvessel endothelial monolayer. Am. J. Physiol. Lung Cell. Mol. Physiol., 284, L187-L196 (2003).

34) Sugita Y, Takao K, Sugino Y, Kuwabara R, Shirahata A. Enhancement of gastrointestinal absorption of ovalbumin caused by spermine induces an increase in plasma histamine levels in mice sensitized to ovalbumin. Biol. Pharm. Bull., 34, 415-419 (2011). 